\title{
Symmetry protected topological phases characterized by isolated exceptional points
}

\author{
S. Lin, L. Jin, ${ }^{*}$ and Z. Song ${ }^{\dagger}$ \\ School of Physics, Nankai University, Tianjin 300071, China
}

(Received 28 November 2017; revised manuscript received 16 April 2019; published 30 April 2019)

\begin{abstract}
Exceptional points (EPs) associated with the coalescence of eigenstates in non-Hermitian systems have many exotic features. The EPs are generally sensitive to system parameters; here we report symmetry protected isolated EPs in the Brillouin zone (BZ) of a two-dimensional non-Hermitian bilayer square lattice. Protected by symmetry, the isolated EPs only move, merge, and split in the BZ. The average values of Pauli matrices under the eigenstate of the system's Bloch Hamiltonian define a real planar vector field, the topological defects of which are isolated EPs associated with vortices. The winding number characterizes the vortices and reveals the topological properties of the non-Hermitian system. Different topological phases correspond to different EP configurations, which are unchanged unless topological phase transition occurs accompanying the EPs merging or splitting.
\end{abstract}

DOI: 10.1103/PhysRevB.99.165148

\section{INTRODUCTION}

Non-Hermitian physics has been extensively investigated in both theoretical and experimental aspects [1-16]. In contrast to phase transition in Hermitian systems, exceptional points (EPs) such as non-Hermitian critical points are associated with the coalescence of eigenstates [17-20]. EPs support many novel phenomena, including power oscillation [21], square-root frequency dependence [22-24], and gap protected long-range entangled state [25]. The eigenstate flips [26-31] and geometric phases imprinted [32-35] when encircling EPs for integer circles reveal the exotic topology of EPs [36-38]. The non-Hermitian topological system enables novel interface states in quasi-one dimension and beyond [39-69]. The topological interface states are classified by two winding numbers originated from the complex magnitude and the varying direction of an effective magnetic field [55]. The Chern number and the vorticity that are associated with the eigenstates and the energy band are topological invariant [70]. For the breakdown of conventional bulk-boundary correspondence, the exotic bulk-edge correspondence is developed [71-74]; the importance of chiral inversion symmetry for the conventional bulk-boundary correspondence is revealed [75]. Non-Hermitian topological phases and EP rings and surfaces protected by symmetries are investigated [76-79]; the important application includes the topological lasing [80-84].

Recently, topological gapless systems, which are related to diabolic points (DPs) [85], have emerged as a new frontier in physics [86-104]. As a joint of two quantum phases, topolog-

\footnotetext{
*jinliang@nankai.edu.cn

${ }^{\dagger}$ songtc@nankai.edu.cn
}

Published by the American Physical Society under the terms of the Creative Commons Attribution 4.0 International license. Further distribution of this work must maintain attribution to the author(s) and the published article's title, journal citation, and DOI. ical gapless systems have band structures with band touching points (BTPs) in the momentum space, where these kinds of nodal points appear as topological defects in an auxiliary vector field $[89,97]$. These points are unremovable due to symmetry protection until a pair of them meet and annihilate together [105]. In general, the EPs of a two-dimensional non-Hermitian system form a loop, referred as an exceptional ring [27,106-108]. A natural question is whether there exist isolated EPs in the momentum space which have similar topological characteristics to nodal points in topological gapless systems.

In this paper, we show that the topological nature of BTPs in the Brillouin zone is determined by the topological defects of a real vector field $\mathbf{F}(\mathbf{k})$ mapped from the Bloch Hamiltonian. $\mathbf{F}(\mathbf{k})$ is composed of the average values of Pauli matrices in non-Hermitian systems, in contrast to an effective magnetic field in Hermitian systems, and a winding number is based on the field $\mathbf{F}(\mathbf{k})$ and characterizes the vortices at EPs as topological charges. Another winding number associated with the energy $\mathbf{E}(\mathbf{k})$ characterizes the topology of band coalescence and the chirality of EPs. Moreover, BTPs are protected by system symmetries and their configurations characterize different topological phases. DPs split into pairs of EPs when non-Hermiticity is introduced; EPs inherit half-integer vortices from their parent DPs. Topological phase transitions occur associated with the creation of new configurations when EPs (DPs) merge or split. The topology of EPs (DPs) is characterized through topological invariants: the winding numbers 0 or $\pm 1 / 2$ $(0$ or \pm 1$)$. Our findings are elucidated through a bilayer square lattice.

\section{BILAYER SQUARE LATTICE}

We consider a tight-binding bilayer square lattice [Fig. 1(a)], typically describing the dissipative ultracold atomic gas in optical lattices [109-114]. The Hamiltonian has 

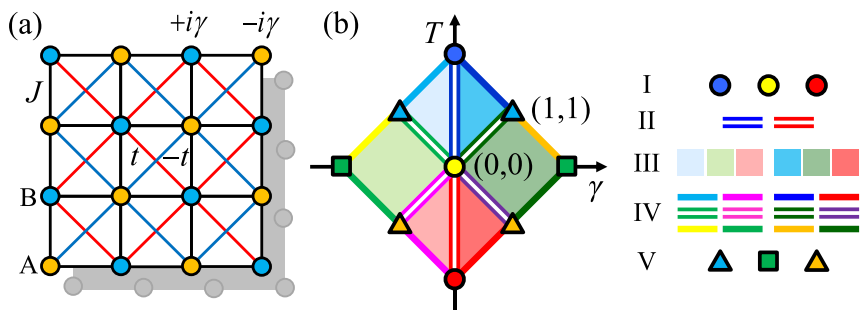

FIG. 1. (a) Schematic of the bilayer square lattice. The intrasublattice hoppings have opposite signs $t$ (red) and $-t$ (blue). The shadowed lattice indicates the lower layer with $(\gamma, t) \rightarrow(-\gamma,-t)$ in contrast to the upper layer. (b) Phase diagram of the bilayer square lattice in the $\gamma-T$ plane for $|T \pm \gamma| \leqslant 2$. Twenty-six topological phases exhibit five types of winding number distributions of BTPs as listed in Table I.

the form $H=\sum_{\lambda=1}^{2} H_{\lambda}+H_{T}$, where the intralayer term is

$$
\begin{aligned}
H_{\lambda}= & \sum_{j, l=1}^{N}[J(|\lambda, j, l\rangle(\langle\lambda, j+1, l|+\langle\lambda, j, l+1|) \\
& \left.+t(-1)^{\lambda+j+l} \sum_{\nu= \pm 1}|\lambda, j, l\rangle\langle\lambda, j+1, l+v|+\text { H.c. }\right] \\
& +i \gamma(-1)^{\lambda+j+l}|\lambda, j, l\rangle\langle\lambda, j, l|,
\end{aligned}
$$

and the interlayer term is $H_{T}=T|1, j, l\rangle\langle 2, j, l|+$ H.c.; $\lambda=$ 1 (2) is the index that labels the upper (lower) layer, and $(j, l)$ is the in-plane site index. In Fig. 1(a), $J$ and $T$ denote the intralayer and interlayer hoppings; $t$ and $\gamma$ are the diagonal couplings and staggered losses. Different sublattices have different losses $\gamma_{\mathrm{A}}$ and $\gamma_{\mathrm{B}}$ due to the environment interactions. After a removal of universal loss $\left(\gamma_{\mathrm{A}}+\gamma_{\mathrm{B}}\right) / 2$, the losses are equivalently described by the balanced gain and loss $\gamma=$ $\left(\gamma_{\mathrm{A}}-\gamma_{\mathrm{B}}\right) / 2$.

The translational symmetry ensures that the Hamiltonian $H$ can be represented as the summation of a series of $2 \times 2$ matrices under the basis $\left|\phi\left(k_{x}, k_{y}\right)\right\rangle_{\mathrm{A}(\mathrm{B})}=$ $\sum_{j, l=1}^{N}(1 / N) e^{i\left(k_{x} j+k_{y} l\right)}\left|\lambda_{\mathrm{A}(\mathrm{B})}, j, l\right\rangle$, where $k_{x}=2 n_{x} \pi / N$ and $k_{y}=2 n_{y} \pi / N$ with $n_{x}, n_{y} \in[1, N]$, and states $\left|\lambda_{\mathrm{A}}, j, l\right\rangle$ and $\left|\lambda_{\mathrm{B}}, j, l\right\rangle$ are the position states of sublattices $A$ and $B$ with layer labels $\lambda_{\mathrm{A}}=\left[3+(-1)^{j+l}\right] / 2$ and $\lambda_{\mathrm{B}}=[3-$ $\left.(-1)^{j+l}\right] / 2$.

The Hamiltonian $H$ in real space is rewritten as the summation of Bloch Hamiltonians $h\left(k_{x}, k_{y}\right)$ in the momentum space:

$$
H=\sum_{k_{x}, k_{y}} h\left(k_{x}, k_{y}\right) \equiv \sum_{k_{x}, k_{y}} \mathbf{B}(\mathbf{k}) \cdot \vec{\sigma}=\sum_{k_{x}, k_{y}} B_{x} \sigma_{x}+B_{y} \sigma_{z} ;
$$

describing an ensemble of noninteracting spin one-half particles in a two-component complex magnetic field is

TABLE I. Five types of BTP distributions.

\begin{tabular}{lccccc}
\hline \hline$w_{\mathrm{I}, \mathrm{II}}$ & I & II & III & IV & V \\
\hline$\{0, \pm 1 / 2, \pm 1\}$ & $\{4,0,0\}$ & $\{0,0,8\}$ & $\{0,16,0\}$ & $\{4,8,0\}$ & $\{8,0,0\}$ \\
\hline \hline
\end{tabular}

$\mathbf{B}(\mathbf{k})=\left(B_{x}, B_{y}, 0\right)$ with

$$
\begin{aligned}
& B_{x}=2 J\left(\cos k_{x}+\cos k_{y}\right)+T, \\
& B_{y}=4 t \cos k_{x} \cos k_{y}+i \gamma,
\end{aligned}
$$

and $\vec{\sigma}=\left(\sigma_{x}, \sigma_{z}, \sigma_{y}\right)$ is a vector of Pauli matrices, with $\sigma_{x}=$ $|\phi\rangle_{\mathrm{A}}\left\langle\left.\phi\right|_{\mathrm{B}}+\mid \phi\right\rangle_{\mathrm{B}}\left\langle\left.\phi\right|_{\mathrm{A}}, \quad \sigma_{y}=-i \mid \phi\right\rangle_{\mathrm{A}}\left\langle\left.\phi\right|_{\mathrm{B}}+i \mid \phi\right\rangle_{\mathrm{B}}\left\langle\left.\phi\right|_{\mathrm{A}}, \quad\right.$ and $\sigma_{z}=|\phi\rangle_{\mathrm{A}}\left\langle\left.\phi\right|_{\mathrm{A}}-\mid \phi\right\rangle_{\mathrm{B}}\left\langle\left.\phi\right|_{\mathrm{B}}\right.$ [115]. The $\mathbf{B}(\mathbf{k})$ field is invariant under interchanging of $k_{x}$ and $k_{y}$. The BTPs are at $\left(k_{\mathrm{c} x}, \pm \pi / 2\right)$ and $\left( \pm \pi / 2, k_{\mathrm{c} y}\right)$, where $\left|k_{\mathrm{c} x(\mathrm{c} y)}\right|=\cos ^{-1}[(-T \pm \gamma) /(2 J)]$; the BTPs are EPs (DPs) for $\gamma \neq 0(\gamma=0)$.

\section{SYMMETRY}

Discrete symmetries play a crucial role in characterizing topological phases [116]. Here, $t$ breaks the inversion symmetry and $\gamma$ breaks the time-reversal symmetry of the bilayer square lattice, but $H$ has a chiral symmetry, $\chi H(J, T, \gamma, t) \chi^{-1}=-H(J, T, \gamma, t)$. The chiral operator $\chi=U C_{4}$ is a combination of a $Z_{2}$ gauge transformation $U$ and a $90^{\circ}$ rotation $C_{4}$. The $Z_{2}$ gauge transformation $U\left|\lambda_{\mathrm{A}(\mathrm{B})}, j, l\right\rangle=(-1)^{\lambda+j+l}\left|\lambda_{\mathrm{A}(\mathrm{B})}, j, l\right\rangle$ changes the system Hamiltonian $H(J, T, \gamma, t)$ into $H(-J,-T, \gamma, t)$. The chiral symmetry instead of time-reversal symmetry or inversion symmetry ensures the Bloch Hamiltonian including only two Pauli matrices [96].

The mirror reflection in the $x$ and $y$ directions $\left(M_{x}\right.$ and $\left.M_{y}\right)$, the translation of one site in the $x$ and $y$ directions $\left(T_{x}\right.$ and $T_{y}$ ), the $90^{\circ}$ rotation $\left(C_{4}\right)$, and the upper and lower layer interchange $(\Lambda)$ all change the Hamiltonian $H(J, T, \gamma, t)$ into $H(J, T,-\gamma,-t)$. Therefore, $H$ is invariant under a combination of any two of the above listed operations and leads to (Appendix A)

$$
h\left(k_{x}, k_{y}\right)=h\left( \pm k_{x(y)}, \pm k_{y(x)}\right) .
$$

Therefore, the number of EPs (DPs) in the bilayer lattice is protected by these symmetries, being invariant unless topological phase transition occurs when EPs (DPs) merge or split in the non-Hermitian (Hermitian) bilayer lattice.

Notably, the non-Hermitian bilayer square lattice $H(J, T, \gamma, t)$ does not have either $\mathcal{P} \mathcal{T}$ symmetry or $\mathcal{C} \mathcal{T}$ symmetry (charge-conjugation symmetry in Ref. [51]) in the situation that $t \neq 0$. The operator $\mathcal{C}$ is defined as $\mathcal{C}\left|\lambda_{\mathrm{A}(\mathrm{B})}, j, l\right\rangle=(-1)^{0(1)}\left|\lambda_{\mathrm{A}(\mathrm{B})}, j, l\right\rangle \quad[117], \quad \mathcal{T}$ is defined as $\mathcal{T} i \mathcal{T}^{-1}=-i$, and $\mathcal{P}$ is defined as $\mathcal{P}\left|\lambda_{\mathrm{A}(\mathrm{B})}, j, l\right\rangle=$ $\left|\lambda_{\mathrm{A}(\mathrm{B})}, N+1-j, l\right\rangle$ or $\left|\lambda_{\mathrm{A}(\mathrm{B})}, j, N+1-l\right\rangle$. In the absence of diagonal coupling $(t=0)$, the system satisfies both $\mathcal{P} \mathcal{T}$ symmetry $\left[(\mathcal{P} \mathcal{T}) H(J, T, \gamma, t)(\mathcal{P} \mathcal{T})^{-1}=H(J, T, \gamma, t)\right]$ and $\mathcal{C} \mathcal{T}$ symmetry $\left[(\mathcal{C} \mathcal{T}) H(J, T, \gamma, t)(\mathcal{C} \mathcal{T})^{-1}=-H(J, T, \gamma, t)\right]$ [117]. $\mathcal{P} \mathcal{T}$ symmetry leads to the spectrum being real or being conjugate pairs. $\mathcal{C T}$ symmetry requires the eigenenergies appearing in pairs with opposite real parts and identical imaginary parts. Thus, the spectrum under $\mathcal{P} \mathcal{T}$ symmetry and $\mathcal{C} \mathcal{T}$ symmetry is purely real or purely imaginary.

The system is inversion symmetric and time-reversal symmetric when $t=\gamma=0$ [70,118]. The inversion symmetry changes the sign of the momentum and interchanges the upper and lower layers. 


\section{TOPOLOGICAL INVARIANTS OF EP}

$h\left(k_{x}, k_{y}\right)$ includes only two Pauli matrices and commutes with the third one, we employ a planar vector field $\mathbf{F}(\mathbf{k})=$ $\left(F_{x}, F_{y}\right)$ to characterize the topology of $h\left(k_{x}, k_{y}\right)$. The zero energy band degeneracy $E_{ \pm}= \pm \sqrt{B_{x}^{2}+B_{y}^{2}}=0$ implies that the expectation value of $h\left(k_{x}, k_{y}\right)$ under its eigenstate is zero, i.e., $\left\langle h\left(k_{x}, k_{y}\right)\right\rangle=B_{x}\left\langle\sigma_{x}\right\rangle+B_{y}\left\langle\sigma_{z}\right\rangle=0$; therefore, $B_{x}=B_{y}=$ $0\left(\left\langle\sigma_{x}\right\rangle=\left\langle\sigma_{z}\right\rangle=0\right)$ at DPs (EPs) in the Hermitian (nonHermitian) system. The expectation values of the two Pauli matrices compose the planar vector field

$$
\mathbf{F}(\mathbf{k})=\left(\left\langle\sigma_{x}\right\rangle,\left\langle\sigma_{z}\right\rangle\right)
$$

for the non-Hermitian system instead of $\mathbf{F}(\mathbf{k})=\left(B_{x}, B_{y}\right)$ for the Hermitian system $[96,97]$. EPs are protected by symmetry, exhibiting behaviors similar to those of DPs in Hermitian lattices and being topologically stable. Moreover, EPs can map to the topological defects of the manifold $\mathbf{F}(\mathbf{k})$, at which $\mathbf{F}(\mathbf{k})$ approaches zero with vortex or antivortex structures.

A topological invariant characterizes the topological property of $\mathbf{F}(\mathbf{k})$,

$$
w_{\mathrm{I}}=\oint_{\mathrm{C}} \frac{d \mathbf{k}}{2 \pi}\left(\hat{F}_{x} \nabla \hat{F}_{y}-\hat{F}_{y} \nabla \hat{F}_{x}\right),
$$

where $\hat{F}_{x(y)}=F_{x(y)} / \sqrt{F_{x}^{2}+F_{y}^{2}}$ and $\nabla=\partial / \partial \mathbf{k}$. The integral is performed in the $k_{x}-k_{y}$ plane along a closed loop $\mathrm{C} ; 2 \pi w_{\mathrm{I}}$ accounts for the varying direction of vector field $\mathbf{F}(\mathbf{k})$. The (both eigenstates yielding identical) winding number $w_{\mathrm{I}}$ describes the vortices with topological charges [70,108] at EPs and is in parallel to the winding number that originated from the generalized Berry phase $[55,103,108]$.

Moreover, the topology of BTP can be determined by winding numbers $w_{\mathrm{I}}$ via the planar vector fields $\mathbf{F}(\mathbf{k})$. By means of $w_{\mathrm{I}}$, the BTP is classified into four types.

(i) Semi-Dirac point. $H(\mathbf{k})$ is Hermitian and the semiDirac point possesses an anisotropic dispersion of linear and quadratic dispersions along two orthogonal directions [119-123]. It has winding number $w_{\mathrm{I}}=0$ and can split into two Dirac points $\left(w_{\mathrm{I}}= \pm 1\right)$ when changing parameters without breaking any symmetries [124].

(ii) Dirac point. $H(\mathbf{k})$ is also Hermitian and the dispersion is linear along all directions around the Dirac point, but the vector field $\mathbf{F}(\mathbf{k})$ owns a vortex $\left(w_{\mathrm{I}}=+1\right)$ or an antivortex $\left(w_{\text {I }}=-1\right)$ with opposite topological charges [125-127]. In the presence of non-Hermiticity, each Dirac point splits into two normal EPs with identical half-integer vortices as in item (iii) [128-130]; in contrast, the Dirac point can split into two Weyl points in Hermitian systems with breaking inversion or time-reversal symmetry [88,93,102,118,131-135].

(iii) Normal EP. The winding numbers of normal EPs are $w_{\mathrm{I}}= \pm 1 / 2$; this indicates that EPs inherit one-half vortices from their parent Dirac points with $w_{\mathrm{I}}= \pm 1$ [96,97]. The dispersion is square root along any direction near the normal EPs.

(iv) Hybrid EP. Two normal EPs with opposite vortices can merge into a hybrid EP. In this situation, the winding number of the EP is changed from $\pm 1 / 2$ to 0 . Different from the dispersion of a semi-Dirac point [119-123], the hybrid EP also has an anisotropic dispersion with square-root (linear) dispersions along two orthogonal directions [70].
While, in parallel to $w_{\mathrm{I}}$, another winding number is defined as

$$
w_{\mathrm{II}}=\oint_{\mathrm{C}} \frac{d \mathbf{k}}{2 \pi}\left(\hat{E}_{x} \nabla \hat{E}_{y}-\hat{E}_{y} \nabla \hat{E}_{x}\right)
$$

with $\mathbf{E}(\mathbf{k})=\left(E_{x}, E_{y}\right)$ being the band energy $E= \pm \sqrt{B_{x}^{2}+B_{y}^{2}}$ in the complex plane, and $\hat{E}_{x}=\operatorname{Re}(E) /|E|$ and $\hat{E}_{y}=$ $\operatorname{Im}(E) /|E|$, which characterizes the topology of EPs on another aspect. Both bands yield identical $w_{\text {II }}$ due to chiral symmetry. $w_{\text {II }}$ differs from $w_{\text {I }}$ and characterizes the topology of band coalescence [55,70], reveals the vorticity of complex Riemann surface band structure [28-30], and reflects the chirality of normal EPs [33-36,136]. Each of the two bands accumulates Berry phase $\pm \pi$ when encircling EPs for two circles; the \pm sign in front determines the opposite chiralities of EPs [19,20,26,33,35] as a result of the square-root-type Riemann sheet. The merging of EPs with opposite chiralities creates a DP or a hybrid EP [36,70]. The winding number is $w_{\mathrm{II}}= \pm 1 / 2$ (0) for normal EPs (DPs and hybrid EPs).

\section{TOPOLOGICAL PHASES}

$H$ is invariant under arbitrary two combinations of the mirror reflection, the translation, the $90^{\circ}$ rotation, and the layer interchange. Symmetries protect the Bloch Hamiltonian $h\left(k_{x}, k_{y}\right)$, being invariant under substitution $\left(k_{x}, k_{y}\right) \rightarrow\left( \pm k_{x(y)}, \pm k_{y(x)}\right)$, and also protect the number of BTPs (DPs and EPs) with vortex structures. The BTPs can move but cannot be removed as system parameters except when they merge or split associated with a topological phase transition and the creation of a new BTP configuration. The BTP configuration (Figs. 2 and 3) characterizes the topological phases of bilayer square lattice, while the phase diagram is depicted in Fig. 1(b).

The system is Hermitian at $\gamma=0$, being time-reversal symmetric without inversion symmetry $(t \neq 0)$. The timereversal symmetry requires DPs at $\pm k$ with identical winding numbers [93,132]. Eight DPs may exist due to the symmetry protection. They locate and move along the $\left|k_{x(y)}\right|=\pi / 2$ lines; their locations satisfy a fourfold rotational symmetry $\left(C_{4}\right.$ symmetry) with respect to the axis that is perpendicular to the $k_{x}-k_{y}$ plane, being mirror reflection symmetric about $k_{x(y)}=0$ and $k_{x}= \pm k_{y}$ [134]. Eight DPs merge into four at $|T|=0$ and $2 J[93,131]$. The top panels of Fig. 2 show the DP configurations.

At $\gamma \neq 0$, each DP splits into 2 normal EPs and the corresponding Bloch Hamiltonian $h\left(k_{x}, k_{y}\right)$ is defective [128-130], generating 16 EPs at maximum. $h\left(k_{x}, k_{y}\right)$ has chiral symmetry, which requires the EPs $\left(E_{ \pm}=0\right)$ on the lines of $k_{x(y)}= \pm \pi / 2$ if $t \neq 0$. Although EPs are independent of $t$, nonzero $t$ is crucial for the existence of isolated EPs. For special system parameters, the hybrid EPs appear at high symmetric points in the Brillouin zone, where normal EPs with the opposite charges merge and the number of EPs reduces.

$w_{\text {I }}$ characterizes the topological features of BTPs as the topological defects of the planar vector field $\mathbf{F}(\mathbf{k})$. The value of $w_{\mathrm{I}}$ is given in Fig. 2. Distinct topological phases are created when BTPs merge or split at topological phase transition. Topological phases being reflection symmetric about $\gamma=0$ 

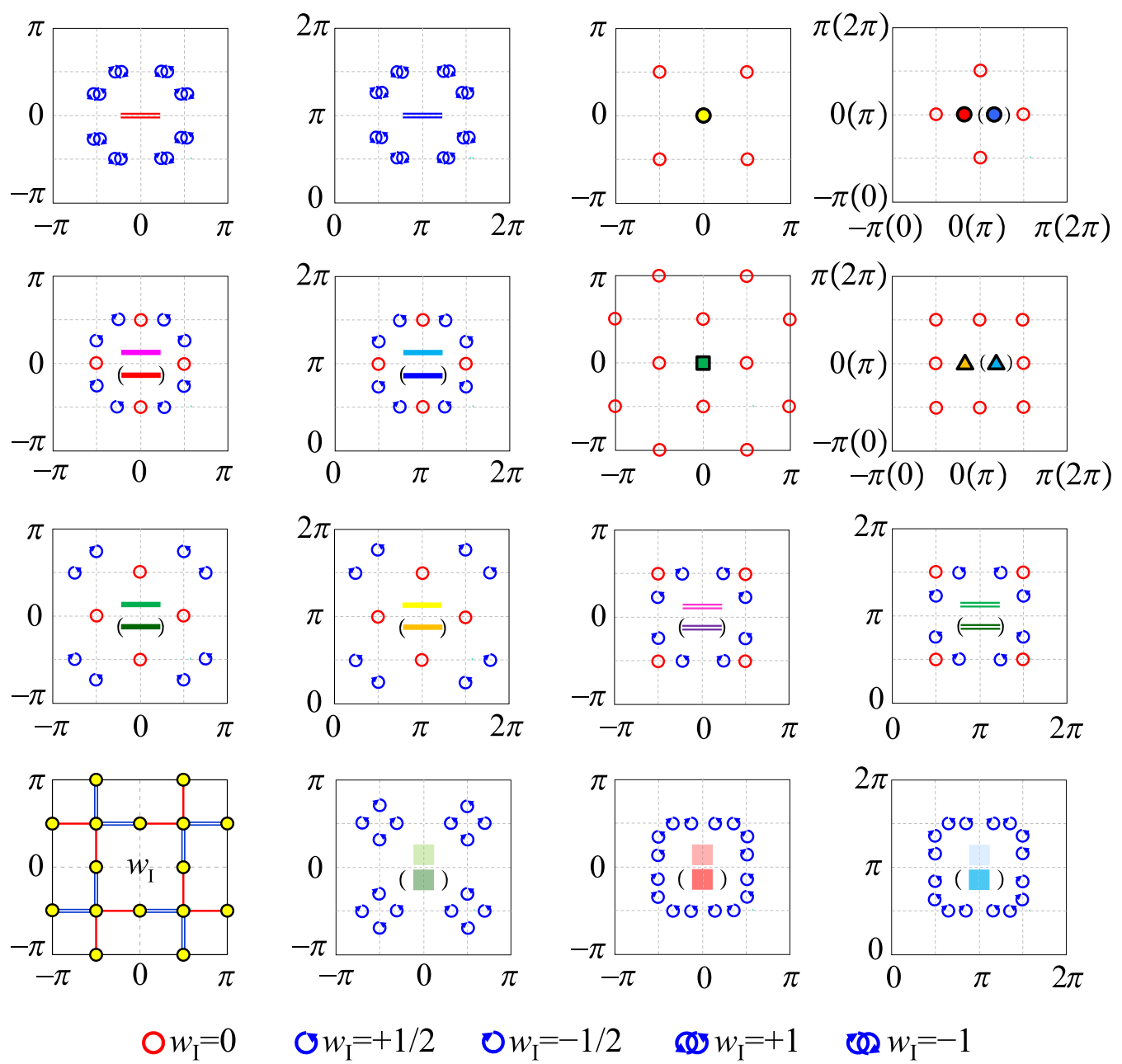

$$
\mathrm{O} w_{\mathrm{I}}=0
$$

$$
\circlearrowleft w_{\mathrm{I}}=+1 / 2 \quad \circlearrowleft w_{\mathrm{I}}=-1 / 2 \quad w_{\mathrm{I}}=+1
$$

ब2 $w_{\mathrm{I}}=-1$

FIG. 2. Schematic of BTP configurations of $w_{\text {I }}$ for the topological phases in Fig. 1(b). The horizontal (vertical) axis is $k_{x}\left(k_{y}\right)$. The blue arrows and red circles represent $w_{\mathrm{I}}= \pm 1 / 2, \pm 1$, and $0 . w_{\mathrm{I}}$ of the Hermitian (non-Hermitian) bilayer lattice is shown in the bottom left corner; the red single line and the blue double lines represent $w_{\mathrm{I}}=+1$ and $-1\left(w_{\mathrm{I}}=+1 / 2\right.$ and $\left.-1 / 2\right)$, and the yellow solid circles represent $w_{\mathrm{I}}=0$ $\left(w_{\mathrm{I}}=0\right)$.

have identical $w_{\text {I }}$ configurations, exhibiting 17 configurations of $w_{\text {I }}$ [Fig. 2].

In Fig. 2, BTPs with $w_{\mathrm{I}}=0$ are semi-Dirac points in the top panels and are hybrid EPs otherwise. The dispersion is linear along the $k_{x(y)}\left(k_{x}= \pm k_{y}\right)$ direction near the semi-Dirac points on the $k_{x(y)}= \pm \pi / 2\left(k_{x}= \pm k_{y}\right)$ line. The dispersion along the $\lambda_{1} k_{x}=\lambda_{2} k_{y}\left(\lambda_{1} k_{x}=-\lambda_{2} k_{y}\right)$ direction is square root (linear) near the hybrid EPs $\left(\lambda_{1} \pi / 2, \lambda_{2} \pi / 2\right)$, where $\lambda_{1,2}=$ \pm 1 . The dispersion along the $k_{x}=0\left(k_{y}=0\right)$ direction is square root (linear) near the hybrid points $(0, \pm \pi / 2)$ and $(\pi, \pm \pi / 2)$.

Three typical types of vortices for the planar vector fields $\mathbf{F}(\mathbf{k})$ and $\mathbf{E}(\mathbf{k})$ in the bilayer lattice are shown in Fig. 4. The EP configurations are depicted, including both the zero and half-integer vortices of $w_{\mathrm{I}, \mathrm{II}}=0$ and $\pm 1 / 2$ for the EPs. Figures 4(a) and 4(b) include 8 one-half vortices (normal EP $w_{\mathrm{I}, \mathrm{II}}= \pm 1 / 2$ ) and 4 zero vortices (hybrid EP $w_{\mathrm{I}, \mathrm{II}}=$ $0)$. The three EPs along $k_{y(x)}=-(+) \pi / 2$ from $k_{x(y)}=-\pi$ to $k_{x(y)}=+\pi$ have $w_{\mathrm{I}}=\{1 / 2,0,-1 / 2\}$; in contrast, $w_{\mathrm{II}}=$ $\{-1 / 2,0,+1 / 2\}$. Figures $4(c)$ and $4(d)$ include 16 one-half vortices (normal EP $w_{\mathrm{I}, \mathrm{II}}= \pm 1 / 2$ ). Figures $4(\mathrm{e})$ and 4(f) include 8 zero vortices (hybrid EP $w_{\mathrm{I}, \mathrm{II}}=0$ ). In essential, the winding numbers $w_{\mathrm{I}}$ and $w_{\mathrm{II}}$ reflect the varying directions of planar vector fields $\mathbf{F}(\mathbf{k})$ and $\mathbf{E}(\mathbf{k})$, respectively; they both capture two topological aspects of EPs and provide an overall perspective of the topological properties of the nonHermitian system. $w_{\text {I }}$ reflects the topological properties of system: EPs naturally inherit fractional topological charges from DPs in the Hermitian situation; $w_{\mathrm{I}}$ is a topological invariant that is equivalent to the winding number originated from the non-Hermitian generalization of the Berry phase that characterizes the varying direction of the effective magnetic field $[55,70,108]$.

$w_{\text {II }}$ characterizes the chirality of EPs. When $\gamma \rightarrow-\gamma$, the band energy vector becomes $\mathbf{E}^{\prime}(\mathbf{k})=\left(E_{x},-E_{y}\right)$ with $w_{\text {II }}(-\gamma)=-w_{\text {II }}(\gamma)$. But $w_{\text {I }}$ is unchanged with $w_{\mathrm{I}}(-\gamma)=$ $w_{\mathrm{I}}(\gamma)$. The phases symmetric about the $T$ axis in the phase diagram [Fig. 1(b)] are two different phases characterized by opposite $w_{\text {II }}$. Thus, all 26 BTP configurations of $w_{\text {II }}$ distinguish the different topological phases. Compared with the BTP configurations of $w_{\text {I }}$, the additional 9 configurations are attributed to the chirality of EPs that are relevant to the positive or negative value of $\gamma$. The BTP configurations of $w_{\text {II }}$ are shown in Fig. 3 as a supplement and a comparison to 


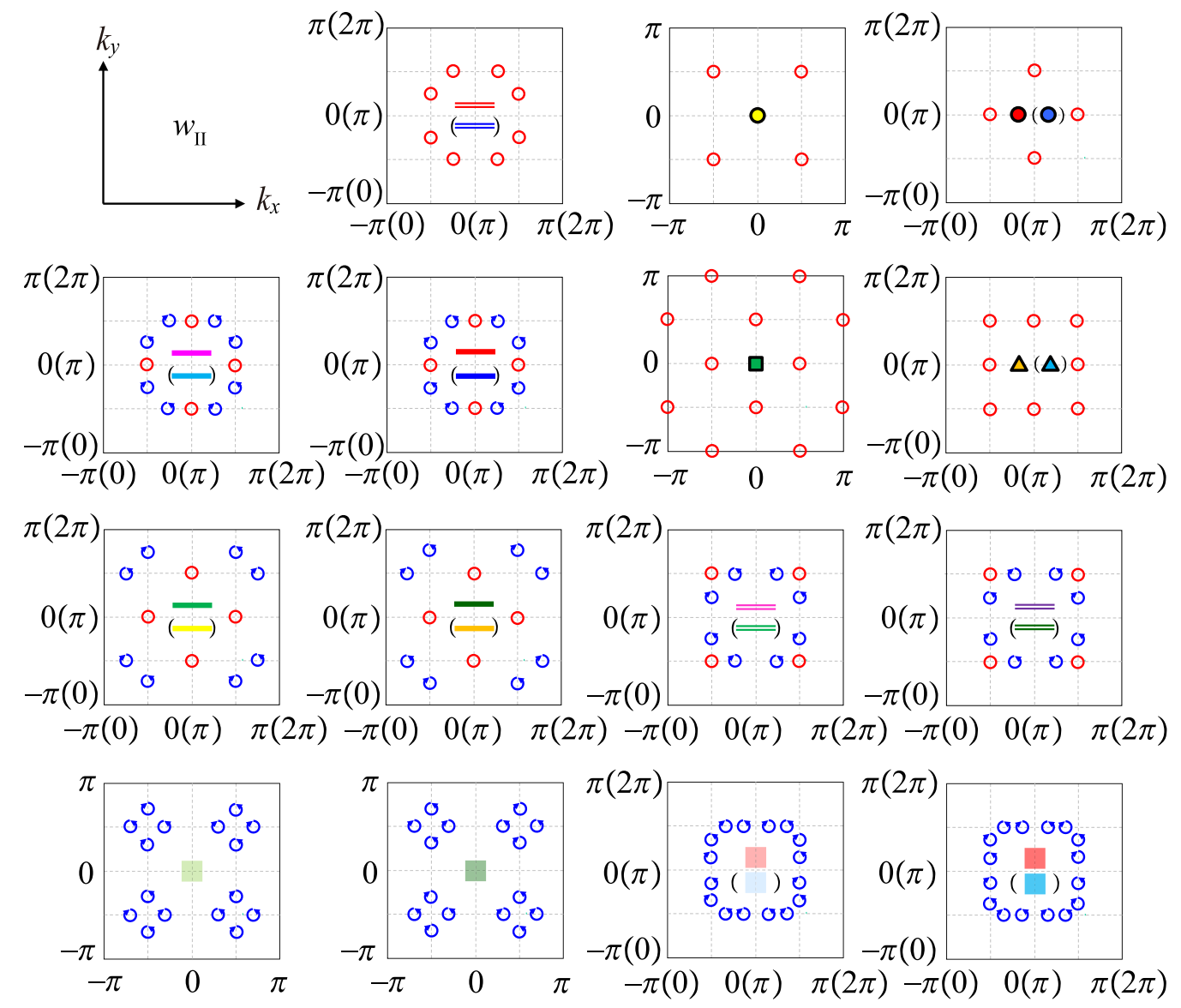

FIG. 3. Schematic of BTP configurations of $w_{\text {II }}$ for the topological phases in Fig. 1(b). The corresponding BTP configurations of $w_{\mathrm{I}}$ are shown in Fig. 2.

the BTP configurations of $w_{\text {I }}$ of Fig. 2. All different phases are distinguishable in the $w_{\text {II }}$ BTP configurations, where the chirality information of EPs is included [18].

The diagonal coupling term $t$ breaks the inversion symmetry and plays a key role in the existence of isolated topologically stable EPs. When $t=0$, the EPs are no longer isolated points but rather rings at $\cos k_{\mathrm{c} x}+\cos k_{\mathrm{cy}}=(-T \pm \gamma) /(2 J)$ [27,76-78,106,107,117,137,138]. However, when $t=0$ and $|\gamma|=|T-4 J|(|T+4 J|)$, the EP rings [27] reduce to isolated points at $\left(k_{\mathrm{c} x}, k_{\mathrm{cy} y}\right)$ of $k_{\mathrm{c} x}=k_{\mathrm{c} y}= \pm \pi(0)$ with trivial topological features $w_{\mathrm{I}}=w_{\mathrm{II}}=0$. The real or imaginary part of spectrum in the momentum space possesses a Dirac-cone band structure for $T>4 J$ or $T<4 J(T<-4 J$ or $T>-4 J)$ at $J>0$; EP vanishes at $|\gamma|>\max \{|T+4 J|,|T-4 J|\}$ or $|\gamma|<\min \{|T+4 J|,|T-4 J|\}$ if $|T|>4 J$. Recently, the topological features of EP rings have been revealed $[108,139]$.

\section{DISCUSSION AND CONCLUSION}

Ultracold atomic gas in optical lattices [109-114,140-147], photonic crystals [148,149], and coupled resonators [55,80,81,130,132,150-153] provide versatile optical platforms for the study of topological physics; the dissipation and radiation in optical systems are non-Hermitian and thus can be considered as possible candidates for the experimental realization of the non-Hermitian bilayer square lattice. The fine-tuned system parameters are particularly beneficial for the realization of topological systems. Several pioneering experimental and theoretical possibilities have been devoted to ultracold atoms in hexagonal and square optical lattices [154-159]. A particular feature of cold atoms in optical lattice is the tunability of the tunneling strengths. One example is the study of topological quantum phase transition in a bilayer square lattice describing ultracold atomic gases in optical lattices. Trapping cold ${ }^{6} \mathrm{Li}$ or ${ }^{40} \mathrm{~K}$ atoms in a dissipative spin-dependent optical lattice is a candidate for realizing the bilayer square lattice [96,108-110,113], and next-nearest-neighbor (diagonal) coupling is introduced by applying additional laser beams. Moreover, the other possible realizations are the coupled resonator waveguide lattice $[55,80,81,130,132,150-153]$ and photonic crystals $[148,149]$. The $\pi$ phase difference between the diagonal couplings $t$ of sublattices $A$ and $B$ is controlled by the optical path length of auxiliary linking resonators (waveguides).

In summary, the symmetry protected isolated EPs in a nonHermitian system are studied, which differ from the symmetry protected EP rings and EP surfaces [76-78]. A real-valued vector field $\mathbf{F}(\mathbf{k})$ is defined by the average values of the Pauli matrix. The topological defects of the field are the BTPs associated with the vortices characterized by the winding number $w_{\text {I }}$, which reflects the topological properties of the system. The EPs own fractional topological charges that are inherited from their parent DPs in Hermitian systems. The type and 

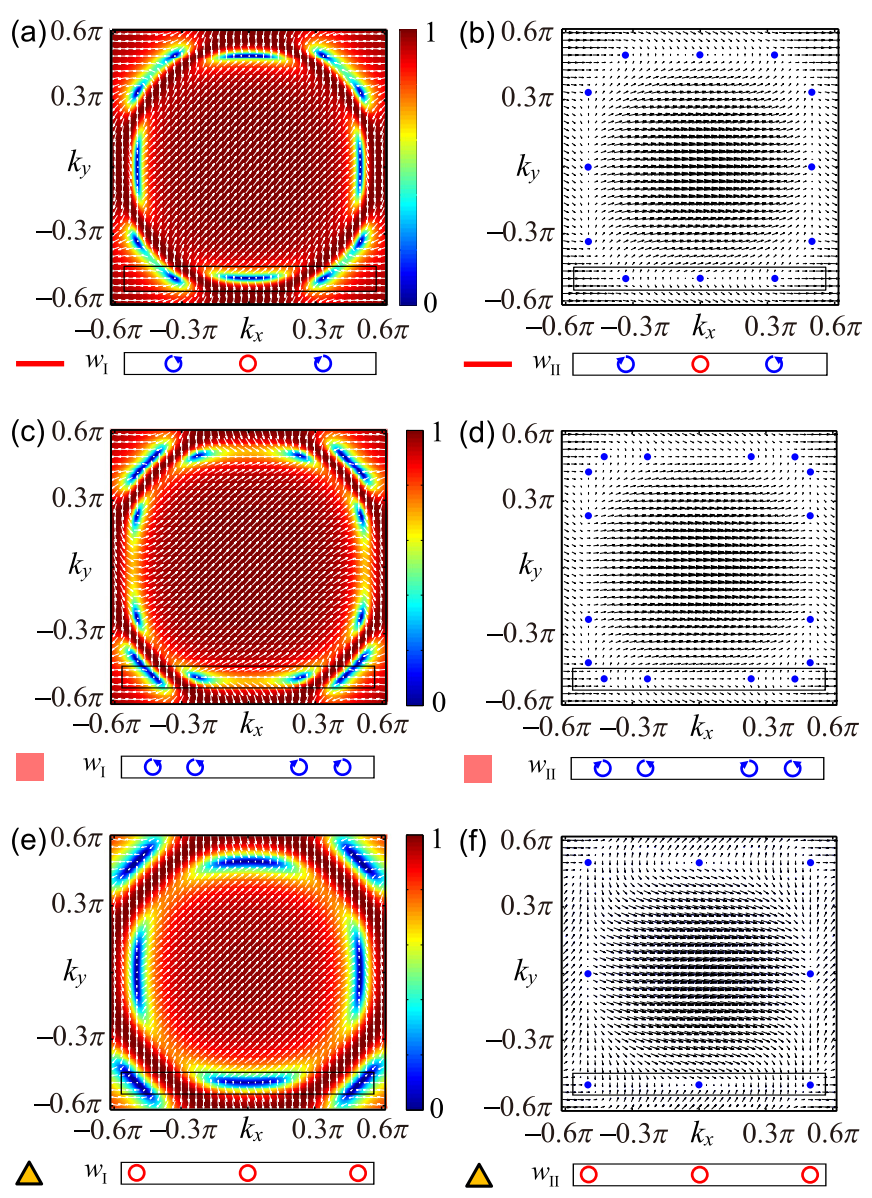

FIG. 4. (a) The planar vector field in the momentum space $\mathbf{F}(\mathbf{k})=\left(F_{x}, F_{y}\right)=\left(\left\langle\sigma_{x}\right\rangle,\left\langle\sigma_{z}\right\rangle\right)$ is shown by the white arrows on the top layer of the left panel, and the background density plot is $F_{x}^{2}+F_{y}^{2}$. (b) $\mathbf{E}(\mathbf{k})=\left(E_{x}, E_{y}\right)=[\operatorname{Re}(E), \operatorname{Im}(E)]$. The black arrows are for $E_{+}$at system parameters $\gamma=J / 2$ and $T=-3 J / 2$. The vortices in the rectangles are indicated by the winding numbers $w_{\text {III })}=+(-)\{+1 / 2,0,-1 / 2\}$ from the left to the right. In panels (c) and (d), the system parameters are $\gamma=J / 2$ and $T=-J$. In panels (e) and (f), $\gamma=T=-J$. The winding numbers of EPs inside the rectangles are schematically illustrated at the bottom of each plot.

number of BTPs are protected by discrete symmetries and only changes at the annihilation or split of the vortex and the antivortex accompanied by a topological phase transition. A pair of half-integer topological charged EPs can merge into a hybrid EP with a vanishing topological charge or a DP with an integer topological charge of zero or one. Another winding number, $w_{\mathrm{II}}$, characterizes the chirality of EPs and the Riemann sheet band structure. $w_{\text {I }}$ and $w_{\text {II }}$ of EPs provide an overall perspective of the topological properties of the nonHermitian system and the BTP configurations outline distinct topological phases. Our findings deepen the understanding of symmetry protected non-Hermitian topological phases and may stimulate interest in searching for topologically stable EPs in other physical systems.

\section{ACKNOWLEDGMENTS}

We acknowledge the support of the National Natural Science Foundation of China (Grants No. 11874225 and No.
11605094), and the Fundamental Research Funds for the Central Universities, Nankai University (Grants No. 63191522 and No. 63191738).

\section{APPENDIX A: SYMMETRY}

The mirror reflection in the $x$ and $y$ directions $\left(M_{x}\right.$ and $\left.M_{y}\right)$, the translation of one site in the $x$ and $y$ directions $\left(T_{x}\right.$ and $\left.T_{y}\right)$, the $90^{\circ}$ degree rotation $\left(C_{4}\right)$, and the upper and lower layer interchange $(\Lambda)$ all change the non-Hermitian bilayer square lattice Hamiltonian $H(J, T, \gamma, t)$ into $H(J, T,-\gamma,-t)$. Therefore, $H$ is invariant under a combination of any two of the above listed operations. For example, the combination operator $\Theta_{1}=M_{x} M_{y}$ leads to $\Theta_{1} H \Theta_{1}^{-1}=H$; under this symmetry operation, the Bloch Hamiltonian in the momentum space changes into $\Theta_{1} h\left(k_{x}, k_{y}\right) \Theta_{1}^{-1}=h\left(-k_{x},-k_{y}\right)$. Another symmetry, $\Theta_{2}=\Lambda M_{x}$, leads to $\Theta_{2} H \Theta_{2}^{-1}=H$; thus, we have $\Theta_{2} h\left(k_{x}, k_{y}\right) \Theta_{2}^{-1}=h\left(-k_{x}, k_{y}\right)$. Similarly, set $\Theta_{3}=\Lambda M_{y}$, and we have $\Theta_{3} h\left(k_{x}, k_{y}\right) \Theta_{3}^{-1}=h\left(k_{x},-k_{y}\right)$; set $\Theta_{4}=M_{x} C_{4}$, and we have $\Theta_{4} h\left(k_{x}, k_{y}\right) \Theta_{4}^{-1}=h\left(k_{y}, k_{x}\right)$; set $\Theta_{5}=M_{y} C_{4}$, and we have $\Theta_{5} h\left(k_{x}, k_{y}\right) \Theta_{5}^{-1}=h\left(-k_{y},-k_{x}\right)$; set $\Theta_{6}=\Lambda C_{4} M_{x} M_{y}$, and we have $\Theta_{6} h\left(k_{x}, k_{y}\right) \Theta_{6}^{-1}=h\left(k_{y},-k_{x}\right)$; and set $\Theta_{7}=C_{2}$, and we have $\Theta_{7} h\left(k_{x}, k_{y}\right) \Theta_{7}^{-1}=h\left(-k_{x},-k_{y}\right)$. These discrete symmetries of the non-Hermitian bilayer square lattice lead to

$$
h\left(k_{x}, k_{y}\right)=h\left( \pm k_{x(y)}, \pm k_{y(x)}\right) .
$$

Therefore, the number of EPs (DPs) in the bilayer lattice is protected by these symmetries, being invariant unless the topological phase transition occurs when EPs (DPs) merge in the non-Hermitian (Hermitian) bilayer lattice.

\section{APPENDIX B: BTP CONFIGURATION}

The complete phase diagram of the bilayer square lattice is illustrated in Fig. 5(a); in contrast to Fig. 1(b), Fig. 5(a) distinguishes all different types of BTP configurations in the system parameter space $\gamma-T$. In Figs. 5(b)-5(e), the system parameters are in the colored areas outside the region $|T \pm \gamma| \leqslant 2$ as a complement of Figs. 2 and 3; notably, all the EPs are still located at $\left(k_{\mathrm{c} x}, \pm \pi / 2\right)$ and $\left( \pm \pi / 2, k_{\mathrm{c} y}\right)$ with $\left|k_{\mathrm{c} x(\mathrm{c} y)}\right|=\cos ^{-1}[(-T \pm \gamma) /(2 J)]$. These topological phases are not discussed in the main text due to the simplicity of this situation. In this situation, eight normal EPs with $w_{\text {I }}= \pm 1 / 2$ exist as shown in the upper panels of Figs. 5(b) and 5(c), and their mergence creates the new configurations as shown in the upper panels of Figs. 5(d) and 5(e), where four hybrid EPs appear. The BTP configurations for $w_{\text {II }}$ outside the region $|T \pm \gamma| \leqslant 2$ are depicted in the lower panels of Figs. 5(b)-5(e).

\section{APPENDIX C: DISPERSION NEAR THE BTPS}

In this Appendix, we discuss the dispersions near the BTPs (EPs and DPs) with different winding numbers. Here we only discuss the dispersion around EPs at $\left(k_{\mathrm{c} x}, \pm \pi / 2\right)$, and the situations of $\left( \pm \pi / 2, k_{\mathrm{c} y}\right)$ can be obtained by interchanging $k_{x}$ and $k_{y}$. Set $q_{x}=k_{x}-k_{\mathrm{c} x}$ and $q_{y}=k_{y}-\pi / 2$, and the Taylor expansion of the $\mathbf{B}(\mathbf{k})$ field to the second order near $\left(k_{\mathrm{c} x}, \pm \pi / 2\right)$ yields

$$
B_{x} \approx 2 J\left[\left(1-q_{x}^{2} / 2\right) \cos k_{c x}-q_{x} \sin k_{c x} \mp q_{y}\right]+T,
$$



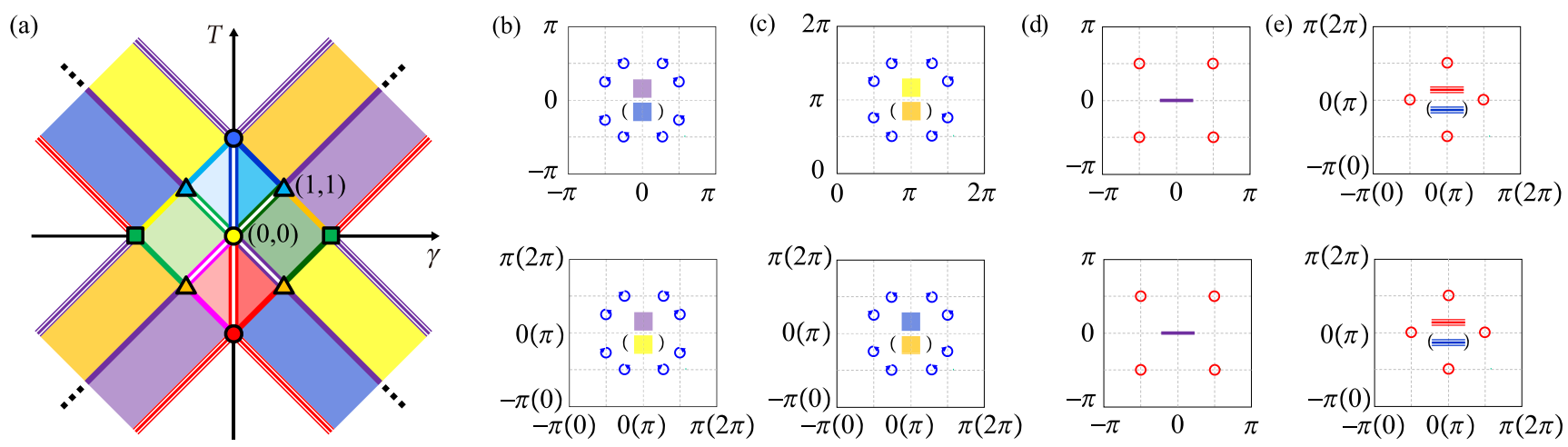

FIG. 5. (a) The phase diagram in the parameter space $\gamma$ - $T$. Sixteen (8) EPs appear in the colored area inside (outside) the region $|T \pm \gamma| \leqslant$ 2 , and the system is gapped in the white region. Except for the entire 26 BTP configurations shown in Figs. 2 and 3, other BTP configurations outside the region $|T \pm \gamma| \leqslant 2$ are illustrated in panels (b)-(e). The upper (lower) panels are the configurations of $w_{\mathrm{I}}\left(w_{\mathrm{II}}\right)$.

$$
B_{y} \approx \mp 4 t q_{y}\left[\left(1-q_{x}^{2} / 2\right) \cos k_{c x}-q_{x} \sin k_{c x}\right]+i \gamma,
$$

and the dispersion is

$$
E_{ \pm}= \pm \sqrt{B_{x}^{2}+B_{y}^{2}}
$$

In the following, we discuss the dispersion near the EPs and DPs in detail.

First, we consider the dispersion near normal EPs with $w_{\mathrm{I}}= \pm 1 / 2$. Near $\left(k_{\mathrm{c} x}, \pm \pi / 2\right)$ with $k_{\mathrm{c} x} \neq 0, \pi$, and $\pm \pi / 2$, we approximately have the dispersion along the $k_{y}=\xi k_{x}$ $\left(k_{x}=-\xi k_{y}\right)$ direction as

$$
\begin{aligned}
& E_{+}\left(k_{y}=\xi k_{x}\right) \\
& \quad \approx 2 \sqrt{-J\left(T+2 J \cos k_{c x}\right)\left(\sin k_{\mathrm{c} x} \pm \xi\right) \mp 2 i \gamma t \xi \cos k_{\mathrm{c} x}} \sqrt{q_{x}}
\end{aligned}
$$

and

$$
\begin{aligned}
& E_{+}\left(k_{x}=-\xi k_{y}\right) \\
& \quad \approx 2 \sqrt{J\left(T+2 J \cos k_{\mathrm{c} x}\right)\left(\xi \sin k_{\mathrm{c} x} \mp 1\right) \mp 2 i \gamma t \cos k_{\mathrm{c} x}} \sqrt{q_{y}},
\end{aligned}
$$

where $\xi$ is real. Notably, the dispersion along any direction is square root near normal EPs with $w_{\mathrm{I}}= \pm 1 / 2$.

Second, we consider the dispersion near hybrid EPs with $w_{\mathrm{I}}=0$. The hybrid points with $w_{\mathrm{I}}=0$ at $k_{\mathrm{c} x}=0, \pi$, and $\pm \pi / 2$ have anisotropic dispersion, being square root in one direction and linear in the perpendicular direction [70]. For $k_{\mathrm{c} x}= \pm \pi / 2$ (i.e., $T \pm \gamma=0$ ), we approximately have the $\mathbf{B}(\mathbf{k})$ field near $\left(\lambda_{1} \pi / 2, \lambda_{2} \pi / 2\right)$ as

$$
B_{x} \approx-2 J\left(\lambda_{1} q_{x}+\lambda_{2} q_{y}\right)+T, \quad B_{y} \approx 4 \lambda_{1} \lambda_{2} t q_{x} q_{y}+i \gamma,
$$

where $\lambda_{1}, \lambda_{2}= \pm$. The dispersion along the $\lambda_{1} k_{x}=\lambda_{2} k_{y}$ $\left(\lambda_{1} k_{x}=-\lambda_{2} k_{y}\right)$ direction is square root (linear) as

$$
\begin{aligned}
& E_{+}\left(\lambda_{1} k_{x}=\lambda_{2} k_{y}\right) \approx 2 \sqrt{-2 \lambda_{1} J T} \sqrt{q_{x}}, \\
& E_{+}\left(\lambda_{1} k_{x}=-\lambda_{2} k_{y}\right) \approx 2 \sqrt{-2 i \gamma t}\left|q_{x}\right| .
\end{aligned}
$$

For $k_{\mathrm{c} x}=0$ (i.e., $T \pm \gamma=-2 J$ ), we approximately have the $\mathbf{B}(\mathbf{k})$ field near $(0, \pm \pi / 2)$ as

$$
B_{x} \approx-J\left(q_{x}^{2} \pm 2 q_{y}-2\right)+T, \quad B_{y} \approx \mp 4 t q_{y}+i \gamma,
$$

and the dispersion along the $k_{x}=0\left(k_{y}=0\right)$ direction is square root (linear) as

$$
\begin{gathered}
E_{+}\left(k_{x}=0\right) \approx 2 \sqrt{\mp J T \mp 2 J^{2} \mp 2 i \gamma t} \sqrt{q_{y}}, \\
E_{+}\left(k_{y}=0\right) \approx \sqrt{-2 J T-4 J^{2}}\left|q_{x}\right| .
\end{gathered}
$$

For $k_{\mathrm{c} x}=\pi$ (i.e., $T \pm \gamma=2 J$ ), we approximately have the $\mathbf{B}(\mathbf{k})$ field near $(\pi, \pm \pi / 2)$ as

$$
B_{x} \approx J\left(q_{x}^{2} \mp 2 q_{y}-2\right)+T, \quad B_{y} \approx \pm 4 t q_{y}+i \gamma,
$$

and the dispersion along the $k_{x}=0\left(k_{y}=0\right)$ direction is square root (linear) as

$$
\begin{gathered}
E_{+}\left(k_{x}=0\right) \approx 2 \sqrt{\mp J T \pm 2 J^{2} \pm 2 i \gamma t} \sqrt{q_{y}}, \\
E_{+}\left(k_{y}=0\right) \approx \sqrt{2 J T-4 J^{2}}\left|q_{x}\right| .
\end{gathered}
$$

Third, the system is Hermitian at $\gamma=0$ and the BTPs are DPs. The dispersion near the DPs with $w_{\mathrm{I}}=0$ is anisotropy, being linear along one direction and quadratic along the perpendicular direction. DPs here are semi-Dirac points [123]. For $k_{c x}= \pm \pi / 2$ (i.e., $T=0$ ), we approximately have the $\mathbf{B}(\mathbf{k})$ field near $\left(\lambda_{1} \pi / 2, \lambda_{2} \pi / 2\right)$ as

$$
B_{x} \approx-2 J\left(\lambda_{1} q_{x}+\lambda_{2} q_{y}\right), \quad B_{y} \approx 4 \lambda_{1} \lambda_{2} t q_{x} q_{y},
$$

and the dispersion along the $\lambda_{1} k_{x}=\lambda_{2} k_{y}\left(\lambda_{1} k_{x}=-\lambda_{2} k_{y}\right)$ direction is linear (quadratic) as

$$
\begin{aligned}
& E_{+}\left(\lambda_{1} k_{x}=\lambda_{2} k_{y}\right) \approx 4 J\left|q_{x}\right|, \\
& E_{+}\left(\lambda_{1} k_{x}=-\lambda_{2} k_{y}\right) \approx 4 t q_{x}^{2} .
\end{aligned}
$$

For $k_{\mathrm{c} x}=0$ (i.e., $T=-2 J$ ), we approximately have the $\mathbf{B}(\mathbf{k})$ field near $(0, \pm \pi / 2)$ as

$$
B_{x} \approx-J\left(q_{x}^{2} \pm 2 q_{y}\right), \quad B_{y} \approx \mp 4 t q_{y},
$$

and the dispersion along the $k_{x}=0\left(k_{y}=0\right)$ direction is linear (quadratic) as

$$
\begin{gathered}
E_{+}\left(k_{x}=0\right) \approx 2 \sqrt{J^{2}+4 t^{2}}\left|q_{y}\right|, \\
E_{+}\left(k_{y}=0\right) \approx J q_{x}^{2} .
\end{gathered}
$$


For $k_{\mathrm{c} x}=\pi$ (i.e., $T=2 J$ ), we approximately have the $\mathbf{B}(\mathbf{k})$ field near $(\pi, \pm \pi / 2)$ as

$$
B_{x} \approx J\left(q_{x}^{2} \mp 2 q_{y}\right), \quad B_{y} \approx \pm 4 t q_{y},
$$

and the dispersion along the $k_{x}=0\left(k_{y}=0\right)$ direction is linear (quadratic) as

$$
\begin{gathered}
E_{+}\left(k_{x}=0\right) \approx 2 \sqrt{J^{2}+4 t^{2}}\left|q_{y}\right|, \\
E_{+}\left(k_{y}=0\right) \approx J q_{x}^{2} .
\end{gathered}
$$

Fourth, we consider the dispersion near DPs with $w_{\mathrm{I}}= \pm 1$. Near $\left(k_{\mathrm{c} x}, \pm \pi / 2\right)$ with $k_{\mathrm{c} x} \neq 0, \pi$, and $\pm \pi / 2$, we approxi- mately have the $\mathbf{B}(\mathbf{k})$ field as

$$
B_{x} \approx 2 J\left(-q_{x} \sin k_{\mathrm{c} x} \mp q_{y}\right), \quad B_{y} \approx \mp 4 t q_{y} \cos k_{\mathrm{c} x},
$$

and the dispersion along the $k_{y}=\xi k_{x}\left(k_{x}=-\xi k_{y}\right)$ direction is

$$
\begin{aligned}
E_{+}\left(k_{y}=\xi k_{x}\right) & \approx 2 \sqrt{J^{2}\left(\sin k_{\mathrm{c} x} \pm \xi\right)^{2}+4 t^{2} \xi^{2} \cos ^{2} k_{\mathrm{c} x}}\left|q_{x}\right|, \\
E_{+}\left(k_{x}=-\xi k_{y}\right) & \approx 2 \sqrt{J^{2}\left(\xi \sin k_{\mathrm{c} x} \mp 1\right)^{2}+4 t^{2} \cos ^{2} k_{\mathrm{c} x}}\left|q_{y}\right| .
\end{aligned}
$$

Notably, DPs with $w_{\mathrm{I}}= \pm 1$ have linear dispersion along any direction; DPs here are Dirac points.
[1] N. Moiseyev, Non-Hermitian Quantum Mechanics (Cambridge University, Cambridge, England, 2011).

[2] C. M. Bender, Rep. Prog. Phys. 70, 947 (2007).

[3] I. Rotter, J. Phys. A 42, 153001 (2009).

[4] W. D. Heiss, J. Phys. A 45, 444016 (2012).

[5] H. Cao and J. Wiersig, Rev. Mod. Phys. 87, 61 (2015).

[6] Z. H. Musslimani, K. G. Makris, R. El-Ganainy, and D. N. Christodoulides, Phys. Rev. Lett. 100, 030402 (2008).

[7] A. Guo, G. J. Salamo, D. Duchesne, R. Morandotti, M. Volatier-Ravat, V. Aimez, G. A. Siviloglou, and D. N. Christodoulides, Phys. Rev. Lett. 103, 093902 (2009).

[8] B. Peng, S. K. Ozdemir, F. Lei, F. Monifi, M. Gianfreda, G. L. Long, S. Fan, F. Nori, C. M. Bender, and L. Yang, Nat. Phys. 10, 394 (2014).

[9] L. Chang, X. Jiang, S. Hua, C. Yang, J. Wen, L. Jiang, G. Li, G. Wang, and M. Xiao, Nat. Photonics 8, 524 (2014).

[10] Z. Zhang, Y. Zhang, J. Sheng, L. Yang, M.-A. Miri, D. N. Christodoulides, B. He, Y. Zhang, and M. Xiao, Phys. Rev. Lett. 117, 123601 (2016).

[11] L. Feng, R. El-Ganainy, and L. Ge, Nat. Photonics 11, 752 (2017).

[12] S. Longhi, Eur. Phys. Lett. 120, 64001 (2017).

[13] Y. Huang, Y. Shen, C. Min, S. Fan, and G. Veronis, Nanophotonics 6, 977 (2017).

[14] L. Jin and Z. Song, Phys. Rev. Lett. 121, 073901 (2018).

[15] R. El-Ganainy, K. G. Makris, M. Khajavikhan, Z. H. Musslimani, S. Rotter, and D. N. Christodoulides, Nat. Phys. 14, 11 (2018).

[16] S. K. Gupta, Y. Zou, X. Y. Zhu, M. H. Lu, L. Zhang, X. P. Liu, and Y. F. Chen, arXiv:1803.00794.

[17] W. D. Heiss and H. L. Harney, Eur. Phys. J. D 17, 149 (2001).

[18] C. Dembowski, H.-D. Gräf, H. L. Harney, A. Heine, W. D. Heiss, H. Rehfeld, and A. Richter, Phys. Rev. Lett. 86, 787 (2001).

[19] C. Dembowski, B. Dietz, H.-D. Gräf, H. L. Harney, A. Heine, W. D. Heiss, and A. Richter, Phys. Rev. Lett. 90, 034101 (2003).

[20] C. Dembowski, B. Dietz, H.-D. Gräf, H. L. Harney, A. Heine, W. D. Heiss, and A. Richter, Phys. Rev. E 69, 056216 (2004).

[21] C. E. Rüter, K. G. Makris, R. El-Ganainy, D. N. Christodoulides, M. Segev, and D. Kip, Nat. Phys. 6, 192 (2010).

[22] J. Wiersig, Phys. Rev. Lett. 112, 203901 (2014).
[23] W. Chen, S. K. Ozdemir, G. Zhao, J. Wiersig, and L. Yang, Nature (London) 548, 192 (2017).

[24] H. Hodaei, A. U. Hassan, S. Wittek, H. Garcia-Gracia, R. ElGanainy, D. N. Christodoulides, and M. Khajavikhan, Nature (London) 548, 187 (2017).

[25] S. Lin, X. Z. Zhang, C. Li, and Z. Song, Phys. Rev. A 94, 042133 (2016).

[26] R. Uzdin, A. Mailybaev, and N. Moiseyev, J. Phys. A 44, 435302 (2011).

[27] B. Zhen, C. W. Hsu, Y. Igarashi, L. Lu, I. Kaminer, A. Pick, S.-L. Chua, J. D. Joannopoulos, and M. Soljačić, Nature (London) 525, 354 (2015).

[28] J. Doppler, A. A. Mailybaev, J. Böhm, U. Kuhl, A. Girschik, F. Libisch, T. J. Milburn, P. Rabl, N. Moiseyev, and S. Rotter, Nature (London) 537, 76 (2016).

[29] H. Xu, D. Mason, L. Jiang, and J. G. E. Harris, Nature (London) 537, 80 (2016).

[30] D. Heiss, Nat. Phys. 12, 823 (2016).

[31] A. U. Hassan, B. Zhen, M. Soljačić, M. Khajavikhan, and D. N. Christodoulides, Phys. Rev. Lett. 118, 093002 (2017).

[32] M. V. Berry, Czech. J. Phys. 54, 1039 (2004).

[33] A. A. Mailybaev, O. N. Kirillov, and A. P. Seyranian, Phys. Rev. A 72, 014104 (2005).

[34] M. Müller and I. Rotter, J. Phys. A 41, 244018 (2008).

[35] S.-D. Liang and G.-Y. Huang, Phys. Rev. A 87, 012118 (2013).

[36] K. Ding, G. Ma, M. Xiao, Z. Q. Zhang, and C. T. Chan, Phys. Rev. X 6, 021007 (2016).

[37] X.-L. Zhang, S. Wang, B. Hou, and C. T. Chan, Phys. Rev. X 8, 021066 (2018).

[38] L. Jin, Phys. Rev. A 97, 012121 (2018).

[39] D. S. Borgnia, A. J. Kruchkov, and R. J. Slager, arXiv:1902.07217.

[40] H. Zhou and J. Y. Lee, arXiv:1812.10490.

[41] M. Ezawa, arXiv:1810.04527; Phys. Rev. B 99, 121411(R) (2019); arXiv:1902.03716.

[42] Y. C. Hu and T. L. Hughes, Phys. Rev. B 84, 153101 (2011).

[43] K. Esaki, M. Sato, K. Hasebe, and M. Kohmoto, Phys. Rev. B 84, 205128 (2011).

[44] G. Q. Liang and Y. D. Chong, Phys. Rev. Lett. 110, 203904 (2013).

[45] H. Schomerus, Opt. Lett. 38, 1912 (2013).

[46] B. Zhu, R. Lü, and S. Chen, Phys. Rev. A 89, 062102 (2014). 
[47] J. M. Zeuner, M. C. Rechtsman, Y. Plotnik, Y. Lumer, S. Nolte, M. S. Rudner, M. Segev, and A. Szameit, Phys. Rev. Lett. 115, 040402 (2015).

[48] C. Yuce, Phys. Lett. A 379, 1213 (2015).

[49] X. Wang, T. Liu, Y. Xiong, and P. Tong, Phys. Rev. A 92, 012116 (2015).

[50] C. Poli, M. Bellec, U. Kuhl, F. Mortessagne, and $\mathrm{H}$. Schomerus, Nat. Commun. 6, 6710 (2015).

[51] S. Malzard, C. Poli, and H. Schomerus, Phys. Rev. Lett. 115, 200402 (2015).

[52] T. E. Lee, Phys. Rev. Lett. 116, 133903 (2016).

[53] C. He, X. C. Sun, X. P. Liu, M. H. Lu, Y. Chen, L. Feng, and Y. F. Chen, Proc. Natl. Acad. Sci. USA 113, 4924 (2016).

[54] S. Weimann, M. Kremer, Y. Plotnik, Y. Lumer, S. Nolte, K. G. Makris, M. Segev, M. C. Rechtsman, and A. Szameit, Nat. Mater. 16, 433 (2017).

[55] D. Leykam, K. Y. Bliokh, C. Huang, Y. D. Chong, and F. Nori, Phys. Rev. Lett. 118, 040401 (2017).

[56] Y.-X. Xiao, G. Ma, Z.-Q. Zhang, and C. T. Chan, Phys. Rev. Lett. 118, 166803 (2017).

[57] L. Jin, P. Wang, and Z. Song, Sci. Rep. 7, 5903 (2017).

[58] L. Jin, Phys. Rev. A 96, 032103 (2017).

[59] M. Klett, H. Cartarius, D. Dast, J. Main, and G. Wunner, Phys. Rev. A 95, 053626 (2017).

[60] S. Lieu, Phys. Rev. B 97, 045106 (2018).

[61] M. Pan, H. Zhao, P. Miao, S. Longhi, and L. Feng, Nat. Commun. 9, 1308 (2018).

[62] W. Zhu, X. Fang, D. Li, Y. Sun, Y. Li, Y. Jing, and H. Chen, Phys. Rev. Lett. 121, 124501 (2018).

[63] J. Hou, Z. Li, X.-W. Luo, Q. Gu, and C. Zhang, arXiv:1808.06972.

[64] Z. Yang and J. Hu, Phys. Rev. B 99, 081102(R) (2019).

[65] H. Wang, J. Ruan, and H. Zhang, Phys. Rev. B 99, 075130 (2019).

[66] K. Kawabata, S. Higashikawa, Z. Gong, Y. Ashida, and M. Ueda, Nat. Commun. 10, 297 (2019).

[67] K. Kawabata, K. Shiozaki, M. Ueda, and M. Sato, arXiv:1812.09133.

[68] Y. Xiong, J. Phys. Commun. 2, 035043 (2018).

[69] C. Yin, H. Jiang, L. Li, R. Lü, and S. Chen, Phys. Rev. A 97, 052115 (2018).

[70] H. Shen, B. Zhen, and L. Fu, Phys. Rev. Lett. 120, 146402 (2018).

[71] S. Yao and Z. Wang, Phys. Rev. Lett. 121, 086803 (2018).

[72] S. Yao, F. Song, and Z. Wang, Phys. Rev. Lett. 121, 136802 (2018).

[73] Z. Gong, Y. Ashida, K. Kawabata, K. Takasan, S. Higashikawa, and M. Ueda, Phys. Rev. X 8, 031079 (2018).

[74] F. K. Kunst, E. Edvardsson, J. C. Budich, and E. J. Bergholtz, Phys. Rev. Lett. 121, 026808 (2018).

[75] L. Jin and Z. Song, Phys. Rev. B 99, 081103(R) (2019).

[76] J. C. Budich, J. Carlström, F. K. Kunst, and E. J. Bergholtz, Phys. Rev. B 99, 041406(R) (2019).

[77] R. Okugawa and T. Yokoyama, Phys. Rev. B 99, 041202(R) (2019).

[78] T. Yoshida, R. Peters, N. Kawakami, and Y. Hatsugai, Phys. Rev. B 99, 121101(R) (2019).

[79] H. Zhou, J. Y. Lee, S. Liu, and B. Zhen, Optica 6, 190 (2019).
[80] H. Zhao, P. Miao, M. H. Teimourpour, S. Malzard, R. ElGanainy, H. Schomerus, and L. Feng, Nat. Commun. 9, 981 (2018).

[81] M. Parto, S. Wittek, H. Hodaei, G. Harari, M. A. Bandres, J. Ren, M. C. Rechtsman, M. Segev, D. N. Christodoulides, and M. Khajavikhan, Phys. Rev. Lett. 120, 113901 (2018).

[82] G. Harari, M. A. Bandres, Y. Lumer, M. C. Rechtsman, Y. D. Chong, M. Khajavikhan, D. N. Christodoulides, and M. Segev, Science 359, eaar4003 (2018).

[83] M. A. Bandres, S. Wittek, G. Harari, M. Parto, J. Ren, M. Segev, D. Christodoulides, and M. Khajavikhan, Science 359, eaar4005 (2018).

[84] P. St-Jean, V. Goblot, E. Galopin, A. Lemaître, T. Ozawa, L. Le Gratiet, I. Sagnes, J. Bloch, and A. Amo, Nat. Photonics 11, 651 (2017).

[85] M. V. Berry and M. Wilkinson, Proc. R. Soc., Ser. A 392, 15 (1984).

[86] X. Wan, A. M. Turner, A. Vishwanath, and S. Y. Savrasov, Phys. Rev. B 83, 205101 (2011).

[87] K. Y. Yang, Y. M. Lu, and Y. Ran, Phys. Rev. B 84, 075129 (2011).

[88] A. A. Burkov and L. Balents, Phys. Rev. Lett. 107, 127205 (2011).

[89] G. Xu, H. Weng, Z. Wang, X. Dai, and Z. Fang, Phys. Rev. Lett. 107, 186806 (2011).

[90] W. Witczak-Krempa and Y. B. Kim, Phys. Rev. B 85, 045124 (2012).

[91] H. Weng, C. Fang, Z. Fang, B. A. Bernevig, and X. Dai, Phys. Rev. X 5, 011029 (2015).

[92] S.-M. Huang, S.-Y. Xu, I. Belopolski, C.-C. Lee, G. Chang, B. K. Wang, N. Alidoust, G. Bian, M. Neupane, C. Zhang, S. Jia, A. Bansil, H. Lin, and M. Z. Hasan, Nat. Commun. 6, 7373 (2015).

[93] S. M. Young, S. Zaheer, J. C. Y. Teo, C. L. Kane, E. J. Mele, and A. M. Rappe, Phys. Rev. Lett. 108, 140405 (2012).

[94] Z. Wang, Y. Sun, X.-Q. Chen, C. Franchini, G. Xu, H. Weng, X. Dai, and Z. Fang, Phys. Rev. B 85, 195320 (2012).

[95] Z. Wang, H. Weng, Q. Wu, X. Dai, and Z. Fang, Phys. Rev. B 88, 125427 (2013).

[96] K. Sun, W. V. Liu, A. Hemmerich, and S. Das Sarma, Nat. Phys. 8, 67 (2012).

[97] J. M. Hou, Phys. Rev. Lett. 111, 130403 (2013).

[98] Z. K. Liu, B. Zhou, Y. Zhang, Z. J. Wang, H. M. Weng, D. Prabhakaran, S.-K. Mo, Z. X. Shen, Z. Fang, X. Dai, Z. Hussain, and Y. L. Chen, Science 343, 864 (2014).

[99] M. Neupane, S.-Y. Xu, R. Sankar, N. Alidoust, G. Bian, C. Liu, I. Belopolski, T.-R. Chang, H.-T. Jeng, H. Lin, A. Bansil, F. Chou, and M. Z. Hasan, Nat. Commun. 5, 3786 (2014).

[100] S.-Y. Xu, I. Belopolski, N. Alidoust, M. Neupane, C. Zhang, R. Sankar, S.-M. Huang, C.-C. Lee, G. Chang, B. K. Wang, G. Bian, H. Zheng, D. S. Sanchez, F. Chou, H. Lin, S. Jia, and M. Z. Hasan, Science 349, 613 (2015).

[101] B. Q. Lv, H. M. Weng, B. B. Fu, X. P. Wang, H. Miao, J. Ma, P. Richard, X. C. Huang, L. X. Zhao, G. F. Chen, Z. Fang, X. Dai, T. Qian, and H. Ding, Phys. Rev. X 5, 031013 (2015).

[102] L. Lu, Z. Wang, D. Ye, L. Ran, L. Fu, J. D. Joannopoulos, and M. Soljačić, Science 349, 622 (2015).

[103] K. Ding, Z. Q. Zhang, and C. T. Chan, Phys. Rev. B 92, 235310 (2015). 
[104] M. Xiao, G. Ma, Z. Yang, P. Sheng Z. Q. Zhang, and C. T. Chan, Nat. Phys. 11, 240 (2015).

[105] J.-M. Hou, Phys. Rev. B 89, 235405 (2014).

[106] A. Szameit, M. C. Rechtsman, O. Bahat-Treidel, and M. Segev, Phys. Rev. A 84, 021806(R) (2011).

[107] A. Cerjan, A. Raman, and S. Fan, Phys. Rev. Lett. 116, 203902 (2016).

[108] Y. Xu, S.-T. Wang, and L.-M. Duan, Phys. Rev. Lett. 118, 045701 (2017).

[109] S. Diehl, A. Micheli, A. Kantian, B. Kraus, H. P. Büchler, and P. Zoller, Nat. Phys. 4, 878 (2008).

[110] F. Verstraete, M. M. Wolf, and J. I. Cirac, Nat. Phys. 5, 633 (2009).

[111] S. Diehl, E. Rico, M. A. Baranov, and P. Zoller, Nat. Phys. 7, 971 (2011).

[112] C.-E. Bardyn, M. A. Baranov, E. Rico, A. İmamoğ lu, P. Zoller, and S. Diehl, Phys. Rev. Lett. 109, 130402 (2012).

[113] C.-E. Bardyn, M. A. Baranov, C. V. Kraus, E. Rico, A. İmamoğlu, P. Zoller, and S. Diehl, New J. Phys. 15, 085001 (2013).

[114] J. C. Budich, P. Zoller, and S. Diehl, Phys. Rev. A 91, 042117 (2015).

[115] Under definitions $\vec{\sigma}^{\prime}=\left(\sigma_{z}, \sigma_{x}, \sigma_{y}\right), \mathbf{B}^{\prime}(\mathbf{k})=\left(B_{x}^{\prime}, B_{y}^{\prime}, 0\right)$ with $B_{x(y)}^{\prime}=B_{y(x)}$, and $\mathbf{F}^{\prime}(\mathbf{k})=\left(\left\langle\sigma_{z}\right\rangle,\left\langle\sigma_{x}\right\rangle\right) ; w_{\mathrm{I}}^{\prime}\left(w_{\mathrm{I}}\right)$ calculated from $\mathbf{F}^{\prime}(\mathbf{k})[\mathbf{F}(\mathbf{k})]$ is consistent with that in Ref. [55] ([96]) and $w_{\mathrm{I}}^{\prime}=-w_{\mathrm{I}}$.

[116] A. P. Schnyder, S. Ryu, A. Furusaki, and A. W. W. Ludwig, Phys. Rev. B 78, 195125 (2008).

[117] S. Lin and Z. Song, Phys. Rev. A 96, 052121 (2017).

[118] L. Fu and C. L. Kane, Phys. Rev. B 76, 045302 (2007).

[119] P. Dietl, F. Piéchon, and G. Montambaux, Phys. Rev. Lett. 100, 236405 (2008).

[120] S. Banerjee, R. R. P. Singh, V. Pardo, and W. E. Pickett, Phys. Rev. Lett. 103, 016402 (2009).

[121] J. Kim, S. S. Baik, S. H. Ryu, Y. Sohn, S. Park, B.-G. Park, J. Denlinger, Y. Yi, H. J. Choi, and K. S. Kim, Science 349, 723 (2015).

[122] L. Du, X. Zhou, and G. A. Fiete, Phys. Rev. B 95, 035136 (2017).

[123] Q. Chen, L. Du, and G. A. Fiete, Phys. Rev. B 97, 035422 (2018).

[124] K. Sun, H. Yao, E. Fradkin, and S. A. Kivelson, Phys. Rev. Lett. 103, 046811 (2009).

[125] G. Montambaux, F. Piéchon, J.-N. Fuchs, and M. O. Goerbig, Phys. Rev. B 80, 153412 (2009).

[126] L.-K. Lim, J.-N. Fuchs, and G. Montambaux, Phys. Rev. Lett. 108, 175303 (2012).

[127] L. Tarruell, D. Greif, T. Uehlinger, G. Jotzu, and T. Esslinger, Nature (London) 483, 302 (2012).

[128] F. Keck, H. J. Korsch, and S. Mossmann, J. Phys. A 36, 2125 (2003).

[129] O. N. Kirillov, A. A. Mailybaev, and A. P. Seyranian, J. Phys. Math. Gen. 38, 5531 (2005).

[130] H. Zhou, C. Peng, Y. Yoon, C. W. Hsu, K. A. Nelson, L. Fu, J. D. Joannopoulos, M. Soljačić, and B. Zhen, Science 359, 1009 (2018).

[131] T. H. Hsieh, H. Lin, J. Liu, W. Duan, A. Bansil, and L. Fu, Nat. Commun. 3, 982 (2012).
[132] Q. Lin, M. Xiao, L. Yuan, and S. Fan, Nat. Commun. 7, 13731 (2016).

[133] S. A. Yang, H. Pan, and F. Zhang, Phys. Rev. Lett. 113, 046401 (2014).

[134] G. B. Halász and L. Balents, Phys. Rev. B 85, 035103 (2012).

[135] B. Q. Lv, N. Xu, H. M. Weng, J. Z. Ma, P. Richard, X. C. Huang, L. X. Zhao, G. F. Chen, C. E. Matt, F. Bisti, V. N. Strocov, J. Mesot, Z. Fang, X. Dai, T. Qian, M. Shi, and H. Ding, Nat. Phys. 11, 724 (2015).

[136] The topological band structure determines the EP chirality, which is relevant to the coalescence eigenstate. In Fig. 4(b), $w_{\text {II }}$ 's from left to right are $+(-)\{-1 / 2,0,+1 / 2\}$ when $\gamma>$ $0(\gamma<0)$; however, the two outer EPs have the identical coalescence state.

[137] Y. Xu and C. Zhang, Phys. Rev. A 93, 063606 (2016).

[138] D.-W. Zhang, Y. X. Zhao, R.-B. Liu, Z.-Y. Xue, S.-L. Zhu, and Z. D. Wang, Phys. Rev. A 93, 043617 (2016).

[139] A. Cerjan, M. Xiao, L. Yuan, and S. Fan, Phys. Rev. B 97, 075128 (2018).

[140] S.-L. Zhu, B. Wang, and L.-M. Duan, Phys. Rev. Lett. 98, 260402 (2007).

[141] I. Bloch, J. Dalibard, and W. Zwerger, Rev. Mod. Phys. 80, 885 (2008).

[142] X.-J. Liu, K. T. Law, T. K. Ng, and P. A. Lee, Phys. Rev. Lett. 111, 120402 (2013).

[143] M. Aidelsburger, M. Atala, M. Lohse, J. T. Barreiro, B Paredes, and I. Bloch, Phys. Rev. Lett. 111, 185301 (2013).

[144] X.-J. Liu, K. T. Law, and T. K. Ng, Phys. Rev. Lett. 112, 086401 (2014).

[145] M. Aidelsburger, M. Lohse, C. Schweizer, M. Atala, J. T. Barreiro, S. Nascimbène, N. R. Cooper, I. Bloch, and N. Goldman, Nat. Phys. 11, 162 (2015).

[146] Z. Wu, L. Zhang, W. Sun, X.-T. Xu, B.-Z. Wang, S.-C. Ji, Y. Deng, S. Chen, X.-J. Liu, and J.-W. Pan, Science 354, 83 (2016).

[147] N. Goldman, J. C. Budich, and P. Zoller, Nat. Phys. 12, 639 (2016).

[148] S. A. Skirlo, L. Lu, Y. Igarashi, Q. Yan, J. Joannopoulos, and M. Soljačić, Phys. Rev. Lett. 115, 253901 (2015).

[149] S. Mittal, S. Ganeshan, J. Fan, A. Vaez, and M. Hafezi, Nat. Photonics 10, 180 (2016).

[150] M. Hafezi, E. A. Demler, M. D. Lukin, and J. M. Taylor, Nat. Phys. 7, 907 (2011).

[151] K. Fang, Z. Yu, and S. Fan, Nat. Photonics 6, 782 (2012).

[152] L. Lu, J. D. Joannopoulos, and M. Soljačić, Nat. Photonics 8, 821 (2014).

[153] D. Leykam, M. C. Rechtsman, and Y. D. Chong, Phys. Rev. Lett. 117, 013902 (2016).

[154] M. A. Baranov, M. Dalmonte, G. Pupillo, and P. Zoller, Chem. Rev. 112, 5012 (2012).

[155] P. Soltan-Panahi, J. Struck, P. Hauke, A. Bick, W. Plenkers, G. Meineke, C. Becker, P. Windpassinger, M. Lewenstein, and K. Sengstock, Nat. Phys. 7, 434 (2011).

[156] T. Graß, R. W. Chhajlany, L. Tarruell, V. Pellegrini, and M. Lewenstein, 2D Mater. 4, 015039 (2017).

[157] C. Gross and I. Bloch, Science 357, 995 (2017).

[158] D. Barredo, V. Lienhard, S. de Léséleuc, T. Lahaye, and A. Browaeys, Nature (London) 561, 79 (2018).

[159] S. Yoo, New J. Phys. 20, 083012 (2018). 